\title{
A Compact Single Layer Reflectarray Antenna Based on Circular Delay-Lines for X-band Applications
}

\author{
Tayyab SHABBIR ${ }^{1}$, Rashid SALEEM ${ }^{1}$, Sabih Ur REHMAN ${ }^{2}$, Muhammad Farhan SHAFIQUE ${ }^{3}$ \\ ${ }^{1}$ Dept. of Telecommunication Engineering, University of Engineering and Technology, Taxila, 47050, Pakistan \\ ${ }^{2}$ School of Computing and Mathematics at Charles Sturt University, Australia \\ ${ }^{3}$ Center for Advanced Studies in Telecommunication, COMSATS Inst. of Information Technology, Tarlai Kalan, \\ Islamabad, 45550, Pakistan
}

\{tayyab.shabbir, rashid.saleem\}@uettaxila.edu.pk, sarehman@csu.edu.au, farhan.shafique@comsats.edu.pk

Submitted September 18, 2017 / Accepted January 8, 2018

\begin{abstract}
This paper presents a compact single layer reflectarray antenna based on a diagonally notched square patch and a pair of circular delay-lines, for X-band applications. The length and width of circular delay-lines are varied and optimized to attain a linear phase range of more than $600^{\circ}$. The effect of incident angle in TE and TM modes at $0^{\circ}, 15^{\circ}$ and $30^{\circ}$ is studied, which offers stable angular phase range. The hybrid Finite Element Boundary Integral (FEBI) method is used for simulation of the whole reflectarray system comprising of $27 \times 27$ elements and being fabricated on a low cost FR-4 laminate. The measured gain of $24.5 \mathrm{dBi}$ with aperture efficiency of $49.5 \%$ is achieved at $10 \mathrm{GHz}$. The proposed design provides the measured 1-dB gain bandwidth of $12.5 \%$ and 3-dB gain bandwidth of $34 \%$. The simulated and measured side-lobe-levels and cross polarizations are less than $-25 \mathrm{~dB}$ and $-40 \mathrm{~dB}$, respectively.
\end{abstract}

\section{Keywords}

Reflectarray antenna, high gain, circular delay-lines, phase range, TE and TM modes, single layer

\section{Introduction}

Traditional high gain antennas like parabolic reflectors and planar phased-arrays are commonly used in long distance wireless communication links. However, these conventional antennas are not suitable for certain applications due to large curved surfaces, complex feed networks and a non-planar topology [1]. Reflectarray antennas have been evolved recently to substitute and alleviate the limitations of conventional antennas. Reflectarray antenna combines the attractive features of parabolic reflectors and planar phased-array [2]. Reflectarrays are low profile, light-weight, cost effective and have less fabrication complexity, owing to their planar layouts. A reflectarray antenna consists of a flat reflecting surface with a number of phase shifting elements and a ground plane on the flip-side [3]. The parasitic array elements offer an ap-

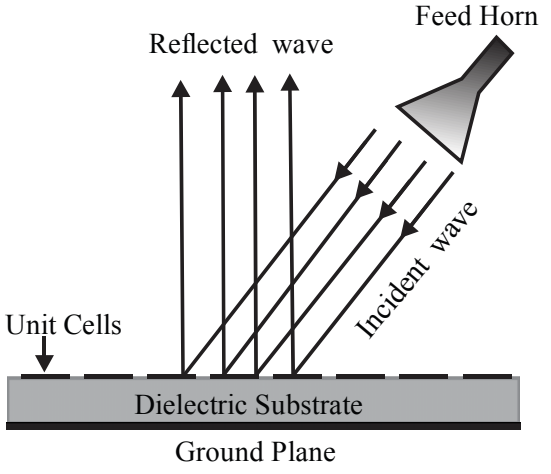

Fig. 1. Reflectarray antenna configuration.

propriate phase shift to generate a focused beam in the desired direction. Reflectarray antenna is usually illumined by a feed horn antenna placed at a focal distance [4]. Conventional reflectarray antenna configuration is depicted in Fig. 1.

Despite advantages, the major shortcoming associated with a reflectarray antenna is its narrow bandwidth. The bandwidth performance of reflectarrays is mostly limited by two factors; narrow-band behavior of radiating elements and spatial phase delays. A narrow-band response is more significant for small to medium-sized reflectarrays. The bandwidth limitations of reflectarray elements can be improved by several techniques, such as by employing multilayer structures, using thicker substrate and connecting phase delay-lines [5], [6]. A linear phase response and improved bandwidth can be obtained by using these techniques. Single layered reflectarrays with delay-lines are reported more since they are broadband, light weight and have higher fabrication tolerances.

In the existing literature, a number of reflectarray configurations are proposed for high gain, linear phase range and stable angular response. The X-band reflectarray antenna with Minkowski radiating element is reported in [7]. A phase range of more than $360^{\circ}$ is achieved by optimizing the width of Minkowski patch. The side-lobe-levels are less than $-18 \mathrm{~dB}$ with HPBW of $7.4^{\circ}$. A single-layer reflectarray with E-shaped element is reported in [8]. The phase range of $360^{\circ}$ is attained by varying the dimensions of 
E-shape element. However, the back lobes and side-lobelevels significantly depend on the width and gap between adjacent E-shape arms. Single-layer reflectarray with combination of elements is presented in [9]. It provides broad reflection phase range by changing the length of outer folded-loop. In [10], a square patch element with attached stubs is proposed for broadband reflectarray. This design provides high gain and a stable angular response. The Xband reflectarray using rectangular-shaped patch element is proposed in [11]. However, phase range is $360^{\circ}$ only and aperture efficiency of $44 \%$ is achieved by changing the grid spacing and element length. A split-ring delay-line configuration is proposed in [12]. The length of delay-line is used to enhance phase range. In [13], a quasi-spiral reflectarray is proposed. The phase range and high gain is achieved by changing the number of spiral turns. The incident angle effect on Transverse Electric (TE), Transverse Magnetic (TM), Feed-to-Diameter (F/D) ratio and phase range is presented in [14]. The microstrip reflectarray with optimized phase range curve is reported in [15]. A gain of $19 \mathrm{dBi}$ is achieved by employing different-sized elements.

In this paper, a compact, single layer, high gain reflectarray is proposed for X-band applications. The proposed design provides broad linear phase range and low cross polarization levels. The X-band reflectarray antenna can be used in aerospace applications, remote sensing and point-to-point communication links. The unit cell comprises of diagonally notched square shape element attached to a pair of circular delay-lines. The proposed design is realized on a low profile FR-4 substrate. A stable angular phase range is observed up to $30^{\circ}$ in TE and TM modes. The reflectarray is designed and optimized by using Ansys High Frequency Structure Simulator $\left(\mathrm{HFSS}^{\mathrm{TM}}\right)$. The simulated and measured results are in good agreement. The rest of the paper is arranged as follows: In Sec. 2, unit cell design and analysis is described. The reflectarray configuration, system simulations and measurements are described in Sec. 3. The paper is finally concluded in Sec. 4.

\section{Unit Element Design and Analysis}

This section describes the reflectarray unit cell configuration, performance parameters and simulation environment setup.

\subsection{Reflectarray Unit Element Configuration}

The reflectarray unit element is designed by making a microstrip square patch which resonates at $10 \mathrm{GHz}$ [16]. The square patch inherently has narrow bandwidth and limited phase range. The elements also have large lattice periodicity and low design efficiency. However, compact single layer elements with large linear phase range are desirable. This has been achieved by adding delay-lines to the square patch. Delay-lines provide broad linear phase range and low cross polarization by varying their length and width [1], [5]. Here, as shown in Fig. 2, a single element is obtained by adding pair of delay-line to a square microstrip patch which is diagonally notched on the four corners to enhance the bandwidth.

The reflectarray is created by periodic arrangement of the reflecting parasitic unit elements along with the ground plane on the flip-side of substrate. The proposed design is etched on $1.6 \mathrm{~mm}$ thick, low cost FR-4 substrate $\left(\varepsilon_{\mathrm{r}}=4.4\right.$, $\tan \delta=0.02)$. The electrical length $\left(\theta_{\mathrm{s}}\right)$ of delay-lines is varied from $0^{\circ}$ to $170^{\circ}$ to control the reflection magnitude and phase range of the reflectarray unit element. The lattice periodicity of elements is kept at $0.25 \lambda_{0}$ at $10 \mathrm{GHz}$ frequency. The proposed reflectarray unit element configuration with side view, TE and TM polarization is shown in Fig. 3.

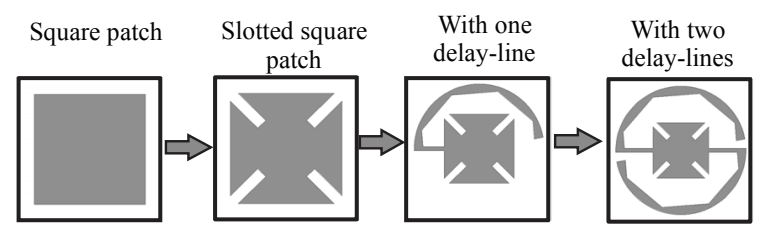

Fig. 2. Design approach of delay-lines based reflectarray.

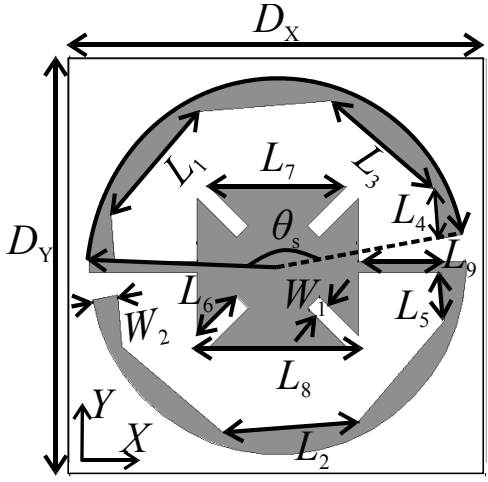

(a) Dimensions

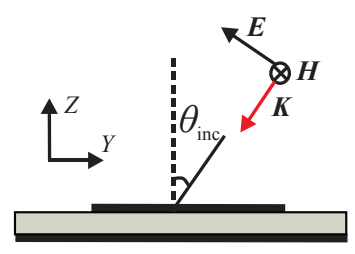

(c) TE polarization

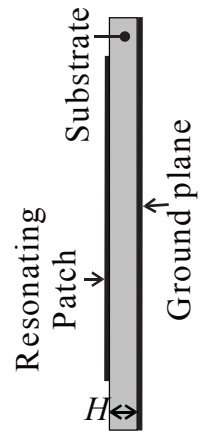

(b) Side view

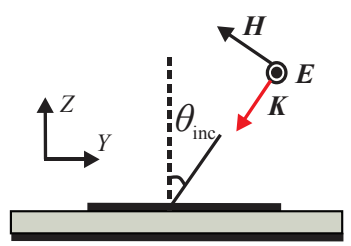

(d) TM Polarization
Fig. 3. Unit cell configuration along with TE and TM mode polarizations

\begin{tabular}{|c|c|}
\hline Parameters & Value $\left(\lambda_{0}\right)$ \\
\hline$D_{\mathrm{X}}$ & 0.25 \\
\hline$D_{\mathrm{Y}}$ & 0.25 \\
\hline$L_{1} L_{2} L_{3}$ & 0.08 \\
\hline$L_{4}, L_{5}$ & 0.03 \\
\hline$L_{6}$ & 0.034 \\
\hline$L_{7}$ & .085 \\
\hline$L_{8}$ & 0.1 \\
\hline$H$ & 0.053 \\
\hline$W_{1}$ & 0.01 \\
\hline$W_{2}$ & 0.016 \\
\hline
\end{tabular}

Tab. 1. The proposed reflectarray unit cell optimized parameters. 


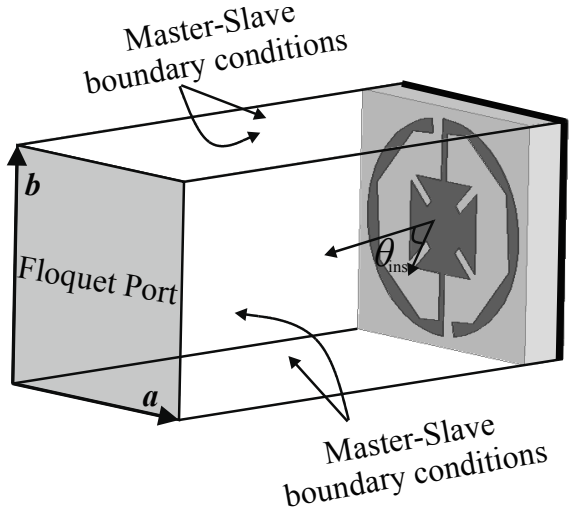

Fig. 4. Reflectarray unit element simulation setup in HFSS.

The reflectarray unit cell optimizations are performed using full-wave simulator with periodic boundary conditions and are excited through a Floquet port setup. Angle $\theta_{\text {ins }}$ is the oblique incidence angle with respect to the normal incidence. The infinite array model is used to calculate the reflection phase response and incident angle effect on the proposed delay-line based structure. Table 1 summarizes different reflectarray parameters.

\subsection{Unit Element Performance Parameters}

The performance of the proposed unit cell is determined in terms of reflection magnitude, phase range and effect of incident angles in TE and TM modes. The phase range and reflection magnitude plot at 9,10 and $11 \mathrm{GHz}$ is shown in Fig. 5. Phase range curves at different frequencies are parallel and vary linearly when delay-lines length $\left(\theta_{\mathrm{s}}\right)$ is varied from $0^{\circ}$ to $170^{\circ}$, which shows the wideband property of reflectarray element. A linear and smooth phase range curve ranging about $600^{\circ}$ is obtained at X-band center frequency of $10 \mathrm{GHz}$.

The TE and TM modes incident angle effect at $0^{\circ}, 15^{\circ}$ and $30^{\circ}$ is shown in Figs. 6(a) and (b). The incident angle has a little influence on the phase range curve of circular delay-line element. The phase range of simple notched square patch is less than $360^{\circ}$. The phase range of reflectarray cell should be greater than $360^{\circ}$ [1]. The phase range plot

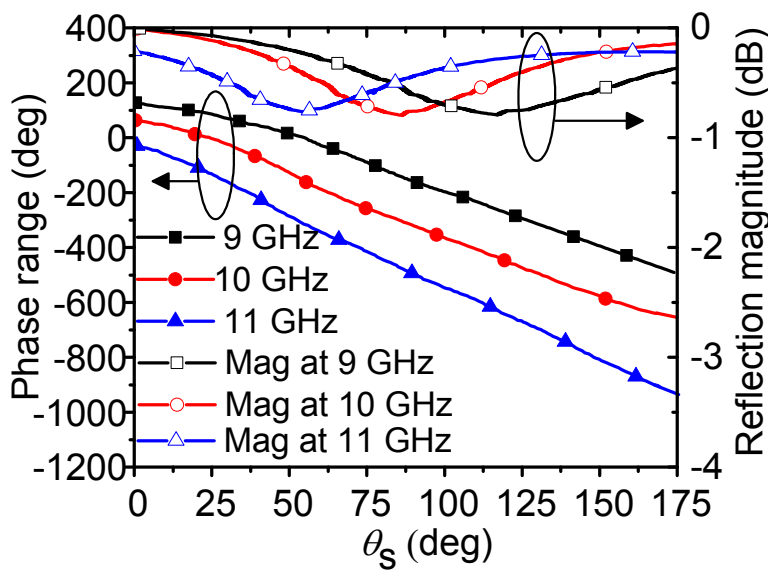

Fig. 5. Reflection magnitude and phase range at 9, 10 and $11 \mathrm{GHz}$, where $\theta_{\mathrm{s}}$ is the delay-line length.

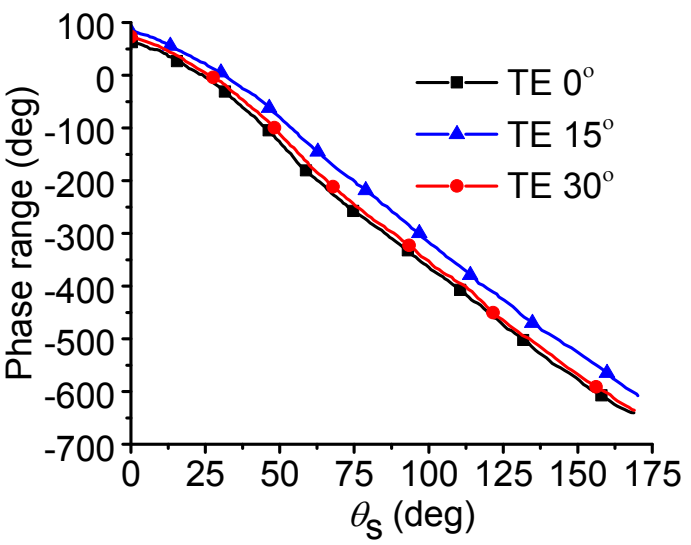

(a) TE mode oblique incidence at $10 \mathrm{GHz}$.

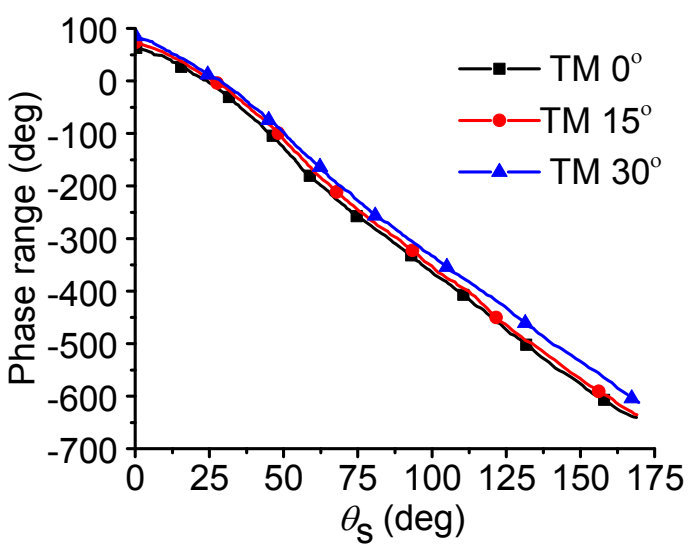

(b) TM mode oblique incidence at $10 \mathrm{GHz}$.

Fig. 6. Reflectarray unit cell TE and TM mode phase range, where $\theta_{\mathrm{s}}$ is the delay-line length.

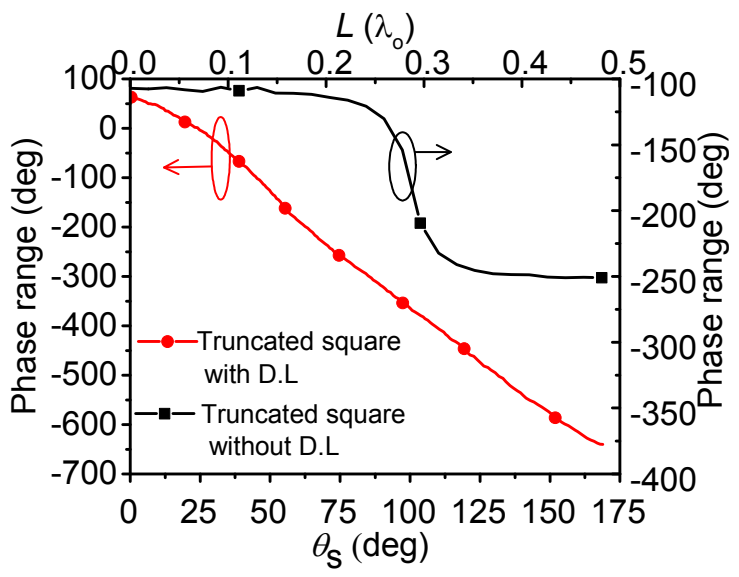

Fig. 7. Phase range with and without delay-lines at $10 \mathrm{GHz}$, where $\theta_{\mathrm{s}}$ is the delay-line length and $L$ is square length.

with and without delay-lines is shown in Fig. 7. A broad linear phase range is achieved by attaching delay-lines to a notched square. The effect of substrate thickness is observed in Fig. 8(a), a linear phase curve behavior is observed for thicker substrate. The effect of different design parameters at $10 \mathrm{GHz}$ is analyzed. By increasing the square slot width and square length, phase curve shifts to lower frequencies, shown in Fig. 8(b). 


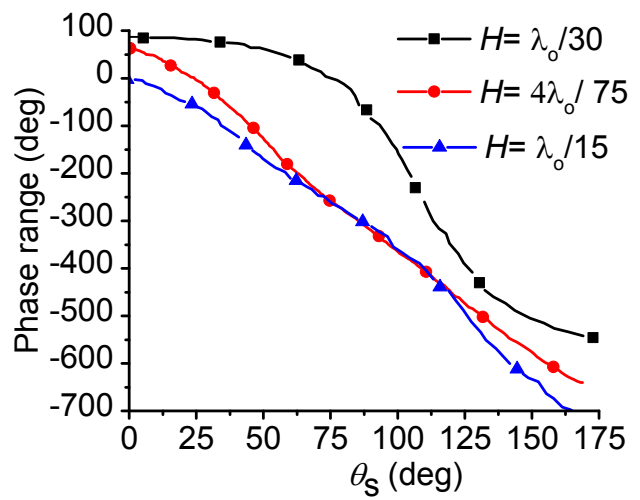

(a)

Effect of substrate thickness at $10 \mathrm{GHz}$, where $H$ is the substrate thickness and $\theta_{\mathrm{s}}$ is the delay-line length.

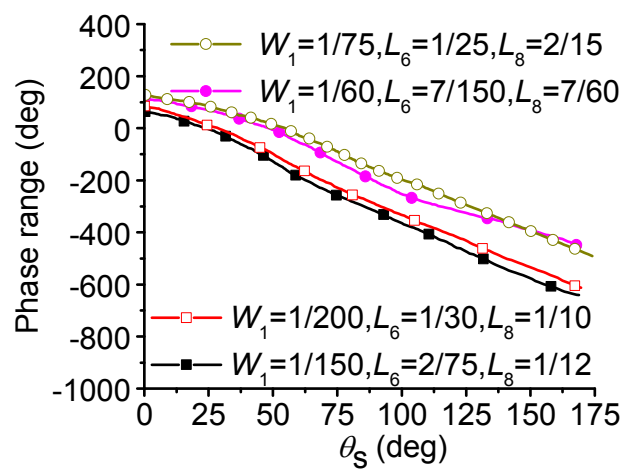

(b) Phase range curve behavior by design parameters, where $W_{1}$ is gap in slotted square, $L_{6}$ is slotted gap length and $L_{8}$ is slotted square width (all parameters in $\left.\lambda_{0}\right)$.

Fig. 8. Parametric analysis at different unit cell parameters.

\section{Full Reflectarray Configuration}

A $27 \times 27$ elements center-fed reflectarray is simulated and measured on a low cost FR-4 substrate. The array elements are etched on the top layer and ground plane is employed at the bottom layer. The distance between array elements is kept at $0.25 \lambda_{0}$ in order to minimize grating lobes [1]. The focal distance $(F)$ and aperture size $(D)$ of the proposed reflectarray are both $6.75 \lambda_{0}$, which indicates the $F / D$ ratio of 1 . The required phase distribution on reflectarray elements can be calculated by using (1). The phase equation compensates the spatial phase delays and provides a focused beam in desired direction at $10 \mathrm{GHz}$.

$$
\varphi_{\mathrm{R}}=k_{0}\left[d_{i}-\left(x_{i} \cos \varphi_{\mathrm{b}}+y_{i} \sin \varphi_{\mathrm{b}}\right) \sin \theta_{\mathrm{b}}\right] .
$$

and

$$
d_{i}=\sqrt{\left(x_{i}-x_{\mathrm{f}}\right)^{2}+\left(y_{i}-y_{\mathrm{f}}\right)^{2}+z_{\mathrm{f}}^{2}}
$$

where $k_{0}$ is free space propagation constant, $d_{i}$ represents the distance between feed horn center and reflectarray $i$ th element, $\left(x_{i}, y_{i}\right)$ is the unit element position and $\left(\theta_{\mathrm{b}}, \phi_{\mathrm{b}}\right)$ the reflectarray main beam direction. The feed horn is placed $6.75 \lambda_{0}$ away for the center-fed arrangement. The phase distribution on reflectarray aperture is shown in Fig. 9. The phase shift plot is used to determine the phase shift value required for each array cell. With the use of phase curve in

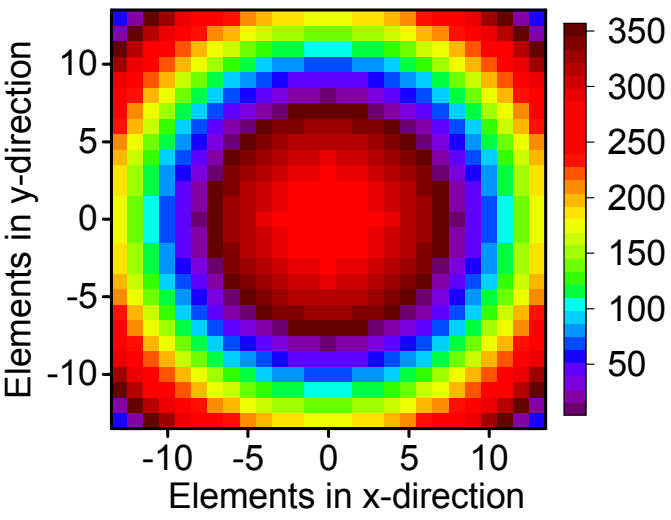

Fig. 9. Phase distribution on reflectarray aperture.

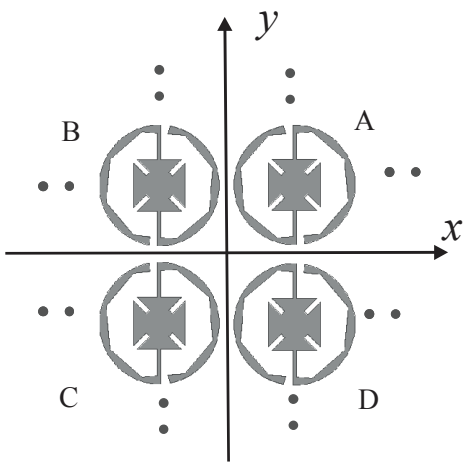

Fig. 10. Four element group arrangement to reduce cross polarization.

Fig. 5, the proposed unit element is configured into $27 \times 27$ elements reflectarray, operating at $10 \mathrm{GHz}$. The circular delay-lines based array elements are placed in mirror arrangement to reduce the cross polarization. The delay-lines based reflectarray is composed of groups of four elements, where each element of a group is the mirror of its neighbor along both $\mathrm{x}$ and $\mathrm{y}$ axis. The four element group is shown in Fig. 10 which is repeated in both $\mathrm{x}$ and $\mathrm{y}$ directions to form a $27 \times 27$ elements reflectarray.

\subsection{FEBI Based System Simulations}

The complete reflectarray system simulations are carried out through hybrid Finite Element Boundary Integral (FEBI) method incorporated in the HFSS software [18]. This method combines the Finite Element Method (FEM) and Integral Equation (IE) and offers faster computation for large sized structures. The 3D-FEBI based reflectarray model is shown in Fig. 11. The feed horn and reflectarray elements are surrounded by two independent radiation boxes. The FEM is applied to the internal structures and IE is assigned to the outer surface of the boxes [14].

The reflectarray model consists of finite number of elements. These elements have different sizes according to phase distribution on array aperture. The radiation patterns computed through FEBI based simulation are more reliable because finite element array configuration is used instead of infinite approximation. The simulation thus caters for the edge effect, incident angles and feed horn position. This method reduces the simulation time and computational re- 


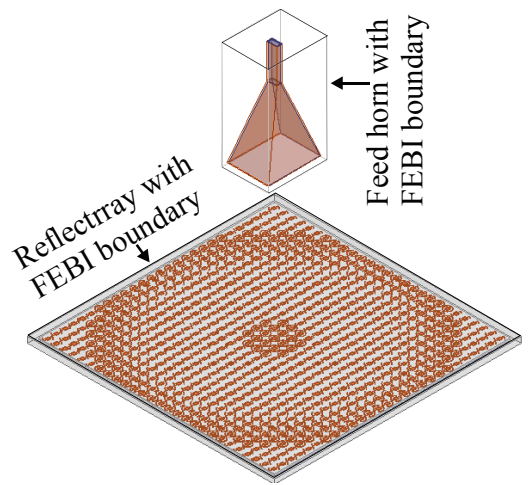

Fig. 11. FEBI based system simulations of actual reflectarray.

sources. An HP Z840 workstation with 64 GB RAM takes about 15 hours for full reflectarray FEBI simulation.

\subsection{Fabrication and Measurement Results}

In order to validate the simulated results, a center-fed reflectarray is fabricated on FR-4 laminate. The proposed unit elements are placed on the top layer with periodicity of $0.25 \lambda_{0}$ and ground plane is placed at bottom layer. The fabricated reflectarray prototype is presented in Fig. 12 (a).

A square wooden stand is manufactured to hold the reflectarray antenna, as shown in Fig. 12(b). The front side is designed to hold the feed horn antenna while reflectarray elements are placed on the rear side of the wooden stand. A Lucas Nuelle ${ }^{\mathbb{R}}$ WR-90 horn antenna is used as feed anten-

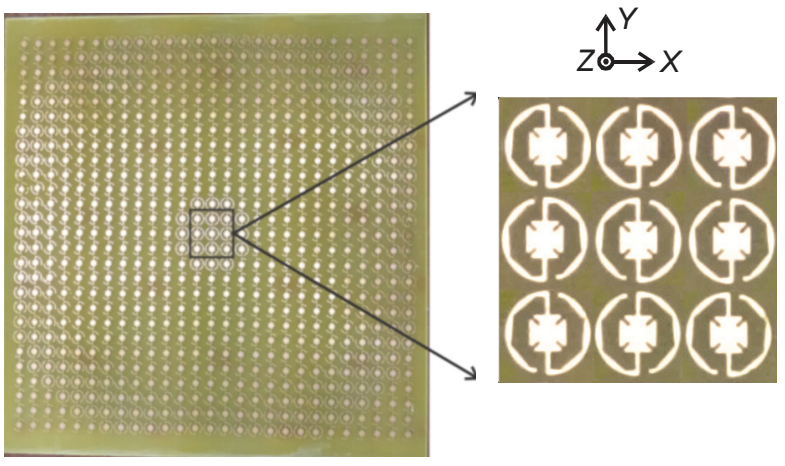

(a) Fabricated reflectarray prototype.

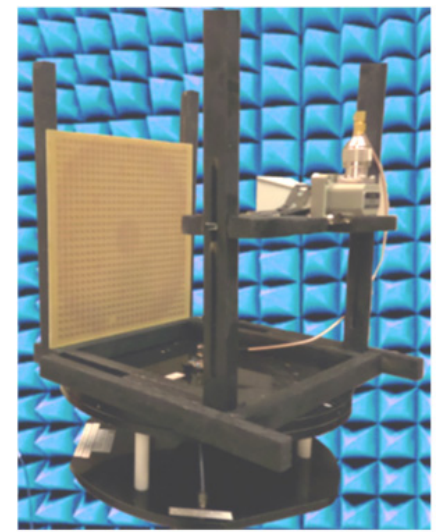

(b) Fabricated reflectarray under testing.

Fig. 12. Reflectarray prototype and testing environment na. The horn antenna operates in X-band (8-12 GHz) with $10 \mathrm{dBi}$ gain. The wooden and Acrylonitrile Butadiene Styrene (ABS) material is used for the testing jig to minimize the reflections in the measurement setup. The reflectarray antenna measurements were made in an anechoic chamber. Agilent N5242A PNA-X network analyzer was used for testing of the proposed reflectarray design. Radiation patterns and gain have been measured using Diamond Engineering setup in anechoic environment.

The proposed reflectarray configuration is measured under normal and oblique incident angles. For the proposed reflectarray configuration, TM and TE mode radiation patterns are similar. Therefore, only TE mode normal and oblique incident angle radiations patterns are included in the manuscript. The normalized simulated and measured far-field radiation patterns of the proposed reflectarray in two major planes (E and $\mathrm{H}$ ) are portrayed in Figs. 13 and 14. The side-lobe-levels are below $-25 \mathrm{~dB}$ in both planes. The highest value of cross-polarization is at least $40 \mathrm{~dB}$ lower than co-polarization at $10 \mathrm{GHz}$ frequency. The simulated and measured half-power beam-width is $15^{\circ}$ at $10 \mathrm{GHz}$.

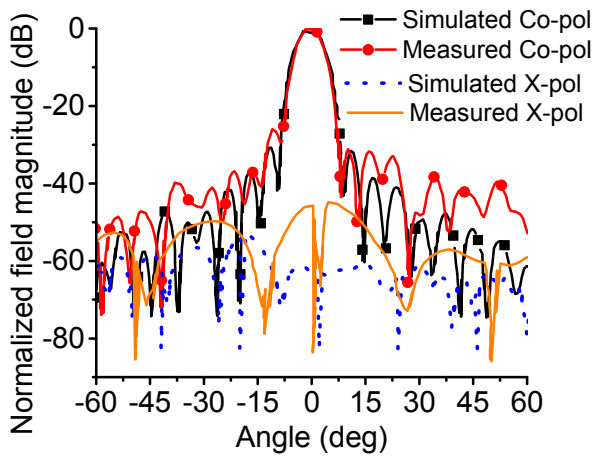

(a) E-plane.

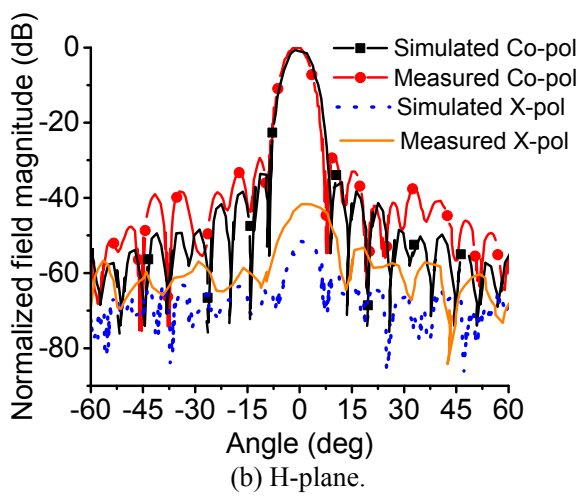

Fig. 13. Normalized radiation patterns under normal incidence at $10 \mathrm{GHz}$ (TE mode)

The simulated and measured gain against frequency is shown in Fig. 15. A gain maximum of $24.5 \mathrm{dBi}$ is achieved at $10 \mathrm{GHz}$ frequency with aperture efficiency of $49.5 \%$. The measured 1-dB gain bandwidth of the proposed reflectarray antenna is $12.5 \%$, in 9.5 to $10.75 \mathrm{GHz}$ band. The $3-\mathrm{dB}$ measured gain bandwidth is $34 \%$, from 8.45 to $11.85 \mathrm{GHz}$. The measured gain is slightly lower than simulated due to the wave blockage effect caused by the support structures of feed horn and measurement system im- 
perfections. The performance of delay-lines reflectarray is better than other reflectarrays in terms of side-lobes-levels, cross polarizations and aperture efficiency. The comparison is shown in Tab. 2.

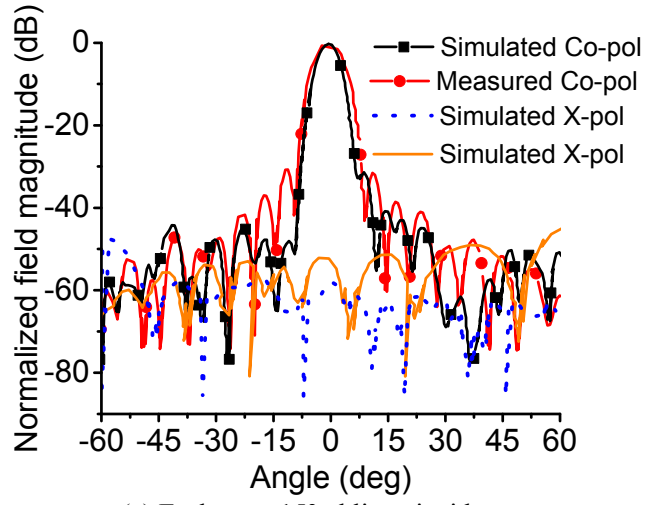

(a) E-plane at $15^{\circ}$ oblique incidence.

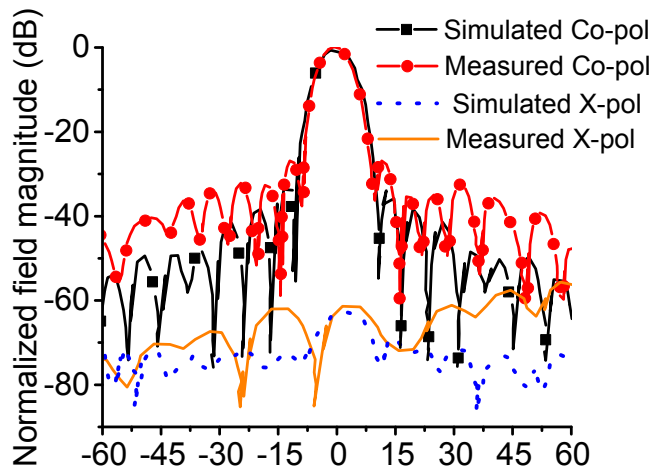

(b) H-plane at $15^{\circ}$ oblique incidence.

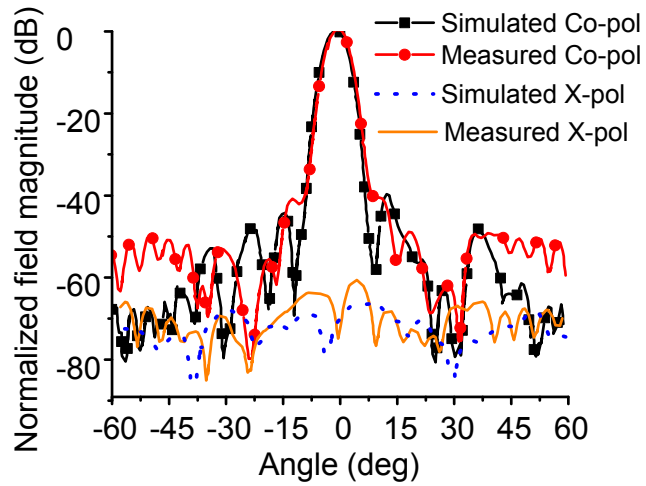

(c) E-plane at $30^{\circ}$ oblique incidence.

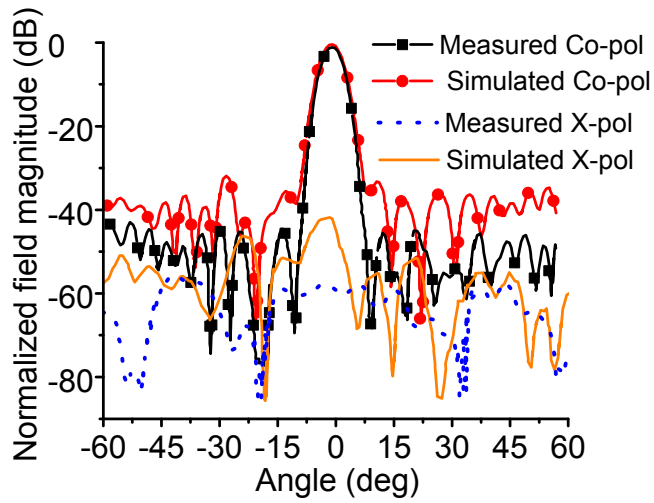

(d) H-plane at $30^{\circ}$ oblique incidence.

Fig. 14. Normalized radiation patterns at $10 \mathrm{GHz}$ under oblique incident angles at $15^{\circ}$ and $30^{\circ}$ (TE mode).

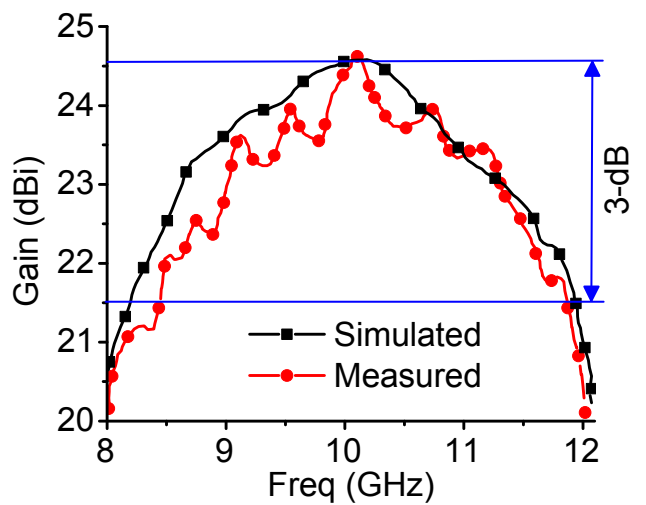

Fig. 15. Gain versus frequency.

\begin{tabular}{|c|c|c|c|c|c|}
\hline Reference No. & $\begin{array}{c}\text { Current } \\
\text { work }\end{array}$ & {$[8]$} & {$[11]$} & {$[15]$} & {$[17]$} \\
\hline $\begin{array}{c}\text { Center } \\
\text { frequency } \\
\text { (GHz) }\end{array}$ & 10 & 7.25 & 10 & 5.8 & 10.2 \\
\hline $\begin{array}{c}\text { Phase shifting } \\
\text { mechanism }\end{array}$ & $\begin{array}{c}\text { Delay- } \\
\text { lines }\end{array}$ & $\begin{array}{c}\text { Length } \\
\text { variation }\end{array}$ & $\begin{array}{c}\text { Size } \\
\text { variation }\end{array}$ & $\begin{array}{c}\text { Different } \\
\text { size } \\
\text { elements }\end{array}$ & $\begin{array}{c}\text { Size } \\
\text { variation }\end{array}$ \\
\hline $\begin{array}{c}\text { 1-dB gain } \\
\text { BW (\%) }\end{array}$ & 12.5 & 8.1 & 18 & - & 26 \\
\hline $\begin{array}{c}3 \text {-dB gain } \\
\text { BW (\%) }\end{array}$ & 34 & 19.8 & - & 12 & 12 \\
\hline Gain (dBi) & 24.5 & 23.7 & 26.1 & 22 & 19 \\
\hline $\begin{array}{c}\text { Aperture } \\
\text { efficiency (\%) }\end{array}$ & 49.5 & 36 & 40.3 & - & 35 \\
\hline F/D & 1 & 0.85 & 1 & - & 0.9 \\
\hline $\begin{array}{c}\text { No. of array } \\
\text { elements }\end{array}$ & 729 & 121 & 729 & 169 & 52 \\
\hline $\begin{array}{c}\text { Unit element } \\
\text { phase range }\end{array}$ & $600^{\circ}$ & $360^{\circ}$ & $325^{\circ}$ & $500^{\circ}$ & $600^{\circ}$ \\
\hline $\begin{array}{c}\text { Cross } \\
\text { polarization } \\
\text { levels (dB) }\end{array}$ & -40 & -20 & -23 & -20 & -21 \\
\hline $\begin{array}{c}\text { Side-lobe- } \\
\text { levels (dB) }\end{array}$ & -25 & - & -16 & -18 & -14 \\
\hline No. of layers & 1 & 1 & 1 & 2 & 2 \\
\hline
\end{tabular}

Tab. 2. Comparison with other related works.

\section{Conclusion}

A single layer reflectarray for X-band applications is presented in this paper. The reflectarray unit cell comprises of diagonally notched square patch attached to circular delay-lines. The unit cell has compact size of $0.25 \lambda_{0}$ designed on low-cost FR-4 substrate. A linear phase range covering $600^{\circ}$ is achieved by simply changing the length of circular delay-lines. The proposed unit cell is angularly stable in TE and TM modes, up to $30^{\circ}$. A $27 \times 27$ elements reflectarray is simulated, fabricated and measured. The reflectarray has Feed-to-Diameter (F/D) ratio of 1 . The measured results shows that $24.5 \mathrm{dBi}$ of gain at $10 \mathrm{GHz}$ is achieved. The simulations and measurements show the proposed antenna has 1-dB bandwidth of $12.5 \%$ from 9.5 to $10.75 \mathrm{GHz}$. The measured cross-polarization and sidelobe-levels are below $-40 \mathrm{~dB}$ and $-25 \mathrm{~dB}$, respectively. The measurement results ensure that the proposed reflectarray is a suitable candidate for high gain X-band applications. 


\section{References}

[1] SHAKER, J., CHAHARMIR, M. R., ETHIER, J. Reflectarray Antennas: Analysis, Design, Fabrication, and Measurement. London (UK): Artech House, 2013. ISBN: 9781608074990

[2] BERRY, D., MALECH, R., KENNEDY, W. The reflectarray antenna. IEEE Transactions on Antennas and Propagation, 1963, vol. 11, no. 6, p. 645-651. DOI: 10.1109/TAP.1963.1138112

[3] DAHRI, M. H., JAMALUDDIN, M. H., ABBASI, M. I., KAMARUDIN, M. R. A review of wideband reflectarray antennas for 5G communication systems. IEEE Access, 2017, vol. 5, p. 17803-17815. DOI: 10.1109/ACCESS.2017.2747844

[4] ROEDERER, G. L. Reflectarray antennas. In The 3rd European Conference on Antennas and Propagation. Berlin (Germany), 2009, p. 18-22.

[5] HAN, C., ZHANG, Y., YANG, Q. A broadband reflectarray antenna using triple gapped rings with attached phase-delay. IEEE Transactions on Antennas and Propagation, 2017, vol. 65, no. 5, p. 2713-2717. DOI: 10.1109/TAP.2017.2679493

[6] HAN, C., ZHANG, Y., YANG, Q. A novel single-layer unit structure for broadband reflectarray antenna. IEEE Antennas and Wireless Propagation Letters, 2016, vol. 16, p. 681-684. DOI: 10.1109/LAWP.2016.2598733

[7] GUNEŞ, F., DEMIREL, S., NESİL, S. Minkowski reflectarray antennas using the full-wave EM simulation-based complete neural model with a hybrid GA-NM algorithm. Radioengineering, 2014, vol. 23, no. 1, p. 144-153. ISSN: 1805-9600.

[8] LEE, S. R., LIM, E. H., LO, F. L. Broadband single-layer E-patch reflectarray. Radioengineering, 2017, vol. 26, no. 1, p. 97-106. DOI: 10.13164/RE.2017.0097

[9] WU, W.-W., QU, S.-W., ZHANG, X.-Q. Single-layer reflectarray with novel elements for wideband applications. Microwave and Optical Technology Letters, 2014, vol. 56, no. 4, p. 950-954. DOI: 10.1002/mop. 28208

[10] QOTOLO, S. F., HASSANI, H. R., NASER-MOGHADASI, M. A novel broadband reflectarray antenna with lattice stubs on square element for Ku-band application. Microwave and Optical Technology Letters, 2015, vol. 57, no. 11, p. 2699-2702. DOI: 10.1002/MOP.29416

[11] WU, J., DA, X., WU, K., et al. Circularly polarized high efficiency wideband reflectarray using rectangle-shaped patch element International Journal of $R F$ and Microwave Computer Aided Engineering, 2017, vol. 27, no. 5. DOI: 10.1002/mmce.21089

[12] NGUYEN, B. D., PHAM, K. T., TRAN, V. S., et al. Reflectarray element using cut-ring patch coupled to delay line. IEEE Antennas and Wireless Propagation Letters, 2015, vol. 14, p. 571-574. DOI: 10.1109/LAWP.2014.2372316

[13] DERAFSHI, I., KOMJANI, N., MOHAMMADIRAD, M. A single-layer broadband reflectarray antenna by using quasispiral phase delay line. IEEE Antennas and Wireless Propagation Letters, 2015, vol. 14, p. 84-87. DOI: 10.1109/LAWP.2014.2355496

[14] ALMAJAli, E. R. F., MCNAMARA, D. A. Angle of incidence effects in reflectarray antenna design: making gain increases possible by including incidence angle. IEEE Antennas and Propagation Magazine, 2016, vol. 58, no. 5, p. 52-64. DOI: 10.1109/MAP.2016.2594699

[15] CHEN, X., CHEN, O., FENG, P., et al. Efficient design of the microstrip reflectarray antenna by optimizing the reflection phase curve. International Journal of Antennas and Propagation, 2016, vol. 2016, 8 p. DOI: 10.1155/2016/8764967

[16] BALANIS. C. A. Antenna Theory: Analysis and Design. $3^{\text {rd }}$ ed New Jersey (U.S): John Wiley \& Sons Inc., 2005. ISBN: 047166782X
[17] ZHAO, J.-J., GONG, S.-X., XU, Y.-X., et al. Design of a broadband reflectarray using meander-shaped elements reflectarray with novel elements for wideband applications. Microwave and Optical Technology Letters, 2012, vol. 54, no. 2, p. 500-503. DOI: $10.1002 /$ mop. 26563

[18] SILVESTRO, J. Hybrid Finite Element Boundary Integral Method. White Paper, 2010, Ansys, Inc., Canonsburg, PA, USA.

\section{About the Authors...}

Tayyab SHABBIR received his B.Sc. degree in Electrical Engineering in 2011 from COMSATS Institute of Information Technology, Islamabad, Pakistan and Masters in Telecommunication Engineering in 2014 from the University of Engineering and Technology (UET) Taxila, Pakistan. In 2014, he was awarded a funded Ph.D. studentship by UET Taxila. His main research interests are in UWBMIMO systems, band-notch antennas, frequency selective surfaces and reflectarrays.

Rashid SALEEM received BS Electronic Engineering from Ghulam Ishaq Khan Institute of Engineering Sciences and Technology, Pakistan, in 1999. He pursued a career in the telecommunication industry for several years while continuing education. He received M.S. from UET Taxila, Pakistan, in 2006 and Ph.D. from The University of Manchester, United Kingdom in 2011. In his PhD, he worked in Microwave and Communication Systems research group under the supervision of Prof. Anthony K. Brown, thenhead of the School of Electrical and Electronic Engineering, The University of Manchester. He worked on antennas, channel modeling and interference aspects of Ultra Wideband systems during his $\mathrm{PhD}$ and was also member of a team designing and testing arrays for the Square Kilometer Array project. Currently, he is working as an Assistant Professor at UET, Taxila, where he is supervising several postgraduate students and heading the MAP (Microwaves, Antennas and Propagation) research group. His research interests include antennas, terahertz communication, microwave periodic structures and metamaterials.

Sabih Ur REHMAN received the bachelor's (Hons.) degree in Electronics and Telecommunication Engineering from the University of South Australia, Adelaide, and the Ph.D. degree in vehicular ad-hoc networks from Charles Sturt University. He has combined experience in the field of information technology/telecommunication of over 16 years in both enterprise and educational sector environments. He is currently a Lecturer in Computing with the School of Computing and Mathematics at Charles Sturt University. His expertise lies in the areas of wireless propagation modeling using mathematical/stochastic models, Ultra Wideband systems, UWB-MIMO antenna systems and band-notch antennas. He is a member of the Institute of Engineers Australia and the Institute of Electrical and Electronic Engineers Computer Society. He was a recipient of a competitive scholarship from Charles Sturt University for his Ph.D

Muhammad Farhan SHAFIQUE received the B.Eng. degree from Hamdard University, Karachi, Pakistan, in 2003, M.S. degree from the University of Paris East 
Marne-La-Vallée, Paris, France, in 2005 and Ph.D. in Electronic and Communications Engineering from The University of Leeds, Leeds, UK in 2010. His research interests involve multilayer microwave device fabrication on LTCC technology, electromagnetic modeling of microwave structures, RF antenna, filters and MEMS packaging. He is also involved in dielectric characterization of materials using microwave techniques and fabrication of ceramic microfluidic devices. He is working as an Associate Director at the Center for Advanced Studies in Telecommunications (CAST) where he has established the MCAD (Microwave Components and Devices) research group. He has also setup a wide range of research facilities in the area of RF engineering which involves 4 state-of-the art laboratories. He is a reviewer of various journals and also a senior member IEEE. 